ORIGINAL

\title{
Androgen replacement therapy for cancer-related symptoms in male advanced cancer patients : study protocol for a randomised prospective trial (ARTFORM study)
}

\author{
Kouji Izumi ${ }^{1}$, Kazuyoshi Shigehara' ${ }^{1}$, Takahiro Nohara ${ }^{1}$, Kazutaka Narimoto', Yoshifumi Kadono ${ }^{1}$, Shigeki Nanjo², \\ Tadaaki Yamada², Koushiro Ohtsubo², Seiji Yano ${ }^{2}$, and Atsushi Mizokami ${ }^{1}$ \\ ${ }^{1}$ Department of Integrative Cancer Therapy and Urology, Kanazawa University Graduate School of Medical Science, Kanazawa, Japan, ${ }^{2}$ Di- \\ vision of Medical Oncology Cancer Research Institute, Kanazawa University, Kanazawa, Japan
}

\begin{abstract}
Recent studies reveal that hypogonadism with low serum androgen levels is associated with advanced cancer and induction of most cancer-related symptoms. We designed an ARTFORM study to evaluate the efficacy of androgen replacement therapy in male advanced cancer patients. The ARTFORM study is an investigatorinitiated, randomised controlled trial comparing intramuscle injection of testosterone enanthate with non-administration in male advanced cancer patients with non-curative locally advanced or metastatic lesions. Serum total and free testosterone levels are measured and patients with low testosterone level are randomised. The primary endpoint is the difference in validated health-related quality of life questionnaires at week 12. Trial registration of the ARTFORM study is assigned to University hospital Medical Information Network, Center identifier UMIN 000010939. J. Med. Invest. 64 : 202 -204, August, 2017
\end{abstract}

Keywords : advanced cancer, androgen replacement therapy, health-related quality of life, randomised controlled trial, testosterone

\section{INTRODUCTION}

Although anticancer agents are sometimes used to prolong survival in advanced cancer patients with non-curative locally advanced or metastatic lesions, many symptoms induced by the cancer itself or the adverse events of anticancer agents burden patients regardless of the type of cancer. Recent studies revealed that hypogonadism with low serum testosterone levels is associated with advanced cancer and induces many cancer-related symptoms including depression, weight loss, albumin loss, sarcopenia, topical inflammation, deterioration of pain, increase of opioid dose, and cachexia (1-5). Currently, each symptom is individually treated with a specific therapy. However, it is theoretically possible to treat hypogonadism by androgen replacement therapy to solve all problems at once. In the ARTFORM study, we prospectively examine the efficacy of androgen replacement therapy for cancerrelated symptoms in male advanced cancer patients.

\section{PATIENTS AND METHODS}

Aim

We aim to evaluate the efficacy of testosterone enanthate in male advanced cancer patients with non-curative locally advanced or metastatic lesions.

\section{Study design}

Our ARTFORM study is an investigator-initiated, randomised controlled trial involving a comparison of intramuscle injection of testosterone enanthate with non-administration in male advanced

Received for publication March 8, 2017 ; accepted March 17, 2017.

Address correspondence and reprint requests to Kouji Izumi, Department of Integrative Cancer Therapy and Urology, Kanazawa University Graduate School of Medical Science, 13-1 Takara-machi, Kanazawa, Ishikawa 920-8641, Japan and Fax : +81-76-234-4263. cancer patients with low possibility of complete recovery. Patients are randomly assigned to testosterone enanthate administration group or non-administration group, as shown in Figure 1.

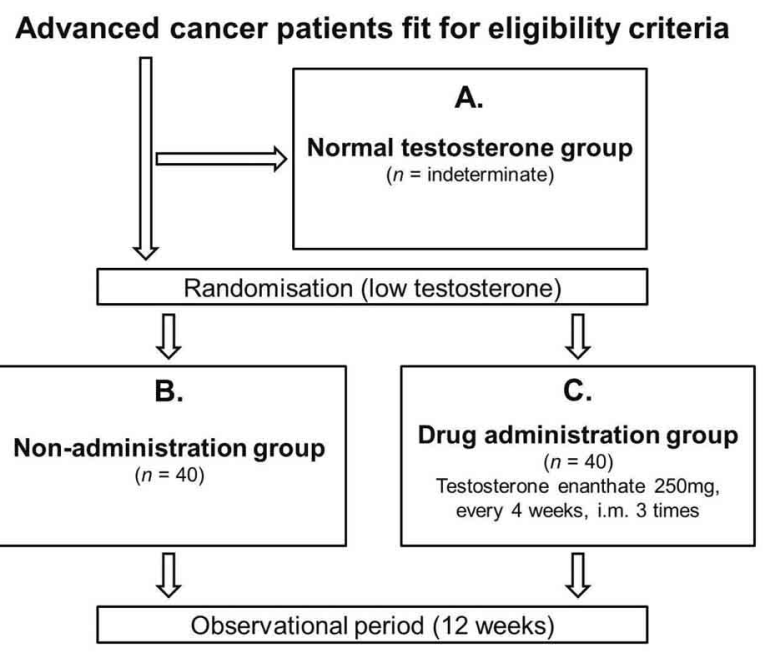

Figure 1. Trial overview

Primary endpoint and additional measures

A validated health-related quality of life questionnaire, FAACT ver4, and the Edmonton Symptom Assessment System (ESAS), translated into Japanese, are administered before the treatment 
and at 4,8 , and 12 weeks after treatment commences to comprehensively evaluate the various aspects of physical and psychosocial well-being.

\section{Eligibility criteria}

Inclusion criteria require patients to be male ; have pathologically or cytologically confirmed cancer; have non-curative locally advanced or metastatic lesions ; know their diagnosis ; provide written informed consent ; and have appropriate hepatic and renal functionality as demonstrated in laboratory tests within 4 weeks prior to registration : aspartate transaminase $\leq 3.0 \times$ upper limit of normal (ULN), alanine transaminase $\leq 3.0 \times \mathrm{ULN}$, blood urea nitrogen $\leq 30 \mathrm{mg} / \mathrm{dL}$, and serum creatinine $\leq 3.0 \mathrm{mg} / \mathrm{dL}$.

Patients are excluded if they are younger than 20 years old ; have prostate cancer, past history of the disease, or severe benign prostate hyperplasia defined as prostate volume $>40 \mathrm{~mL}$ with a history of acute urinary retention ; have possible prostate cancer with serum prostate specific antigen level $>4.0 \mathrm{ng} / \mathrm{mL}$; or are considered by a principal or clinical investigator to be inappropriate for participation in the present study for any other reason.

\section{Informed consent : Ethics approval}

The ARTFORM study is conducted in accordance with the Declaration of Helsinki 1975, as revised in 2008. All treatments and examinations for hypogonadism are undertaken following written informed consent prior to registration. The ARTFORM study received approval from the Institutional Review Board of Kanazawa University.

\section{Methods of recruitment and randomisation}

Recruitment began in June 2013 and completion is planned by December 2017. Eligible patients have their serum total and free testosterone levels checked and if the total and free testosterone level is $\geq 2.31 \mathrm{ng} / \mathrm{mL}$ and $11.8 \mathrm{pg} / \mathrm{mL}$, respectively, patients are assigned to group $\mathrm{A}$ and do not proceed to randomisation. If either total or free testosterone level is $<2.31 \mathrm{ng} / \mathrm{mL}$ or $<11.8 \mathrm{pg} / \mathrm{mL}$, respectively, patients are randomly assigned to one of two groups : non-administration (group B) or testosterone enanthate administration (group C).

\section{Administration of testosterone enanthate}

Testosterone enanthate is injected in muscle of patients who are assigned to the testosterone enanthate group at a dose of $250 \mathrm{mg}$ every 4 weeks. The administration of testosterone enanthate is terminated when any of the following events occur : 1) the patient withdraws written informed consent or opts out of the study;2) the patient is no longer eligible for the study ; 3 ) cancer is completely cured ; 4) the patient's condition deteriorates and testosterone enanthate is not appropriate ; 5) severe adverse events occur; 6) the patient's compliance is poor ; or 7) the patient's participation is considered inappropriate by a principal or clinical investigator for any other reason.

\section{Data collection}

All patients providing written informed consent to participate in the ARTFORM study are asked to provide a complete medical history. Clinical data obtained in the ARTFORM study include the Eastern Cooperative Oncology Group PS, physical measurements (age, height, body weight, body temperature, and blood pressure), haematological measurements (e.g. white blood cell, red blood cell, haemoglobin, haematocrit, and plateletcounts), general blood biochemical measurements (e.g. total and free testosterone, alkaline phosphatase, bone alkaline phosphatase, total bilirubin, creatinine, liver enzymes, and electrolytes), cachexia-related biomarkers (IL-6, TNF alpha, IGF-1), urine analysis, and quality of life questionnaires (FAACT ver4 and ESAS). Examinations are per- formed every 4 weeks for 12 weeks (Figure 2). However, if a principal or clinical investigator considers these examinations to be necessary, they can be performed at any time.

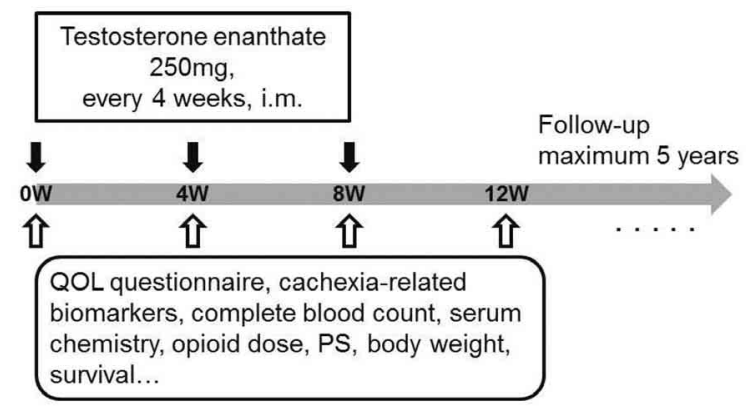

Figure 2. A follow-up schedule

\section{Definition of endpoints}

The primary endpoint is the difference in health-related quality of life questionnaires between groups B and C at week 12 .

Secondary endpoints are set in the ARTFORM study as follows :

1. Difference in health-related quality of life questionnaires, PS, body weight, body mass index, dose of analgesic, and blood data at registration between normal testosterone group (A) and low testosterone groups (B and C).

2. Difference in PS, body weight, body mass index, dose of analgesic, blood data, cancer-specific survival, and overall survival between groups $\mathrm{B}$ and $\mathrm{C}$.

\section{RESULTS}

Patient enrolment and anticipated completion of enrolment

Our current expectation is that the final patient will be enrolled by October 2017 and the entire study will be completed by December 2017. Recruitment began in June 2013 and cumulative enrolment reached 70 cases as of February 2017.

\section{DISCUSSION}

Recent developments in cancer treatment have prolonged survival of advanced cancer patients, indicating that patients spend a long time not only with cancer but also cancer-related symptoms. Although prolonging life is the main goal of cancer treatment, health-related quality of life should also be considered during the period of anticancer treatment and best supportive care. Among male advanced cancer patients with cachexia, up to $70 \%$ of patients reportedly have hypogonadism defined as low serum testosterone levels which could induce many cancer-related symptoms including cachexia itself $(1,2,6)$. Testosterone functions to keep males healthy and decreases gradually with age $(7,8)$. Testosterone increases bone mineral density and muscle mass $(9,10)$, improves mental function and cognitive impairment $(11,12)$, decrease lower urinary tract symptoms and nocturia $(13,14)$, and maintains cardiovascular health (15). On the other hand, androgen receptors in 
prostate cancer cells are important drivers of cancer progression and testosterone may be associated with carcinogenesis of prostate cancer and deteriorate benign prostate hyperplasia (16-18).

Although androgen replacement therapy reportedly improves symptoms caused by hypogonadism in non-cancer male patients, there are few studies showing the efficacy of androgen replacement therapy on symptoms in male advanced cancer patients (19). There have been no prospective studies comparing continuous testosterone administration to non-administration conducted to date. The ARTFORM study is the first study of its kind, and the efficacy of androgen replacement therapy is prospectively assessed from a patient perspective of direct clinical benefit using health-related quality of life questionnaires comparing testosterone administration patients to non-administration patients. The study will clarify the role of testosterone in male advanced cancer patients and the efficacy of androgen replacement therapy on cancer-related symptoms.

\section{$\mathrm{COI}$}

None

\section{ACKNOWLEDGEMENTS}

We thank Maki Morita for data collection.

\section{REFERENCES}

1. Del Fabbro E, Hui D, Dalal S, Dev R, Nooruddin ZI, Bruera E : Clinical outcomes and contributors to weight loss in a cancer cachexia clinic. J Palliat Med 14 : 1004-1008, 2011

2. Strasser F, Palmer JL, Schover LR, Yusuf SW, Pisters K, Vassilopoulou-Sellin R, DeGracia B, Willey JS, Bruera E : The impact of hypogonadism and autonomic dysfunction on fatigue, emotional function, and sexual desire in male patients with advanced cancer : a pilot study. Cancer 107 : 2949-2957, 2006

3. Chlebowski RT, Heber D : Hypogonadism in male patients with metastatic cancer prior to chemotherapy. Cancer Res 42 : 2495-2498, 1982

4. Garcia JM, Li H, Mann D, Epner D, Hayes TG, Marcelli M, Cunningham GR : Hypogonadism in male patients with cancer. Cancer 106 : 2583-2591, 2006

5. Akamizu T, Kangawa K: Ghrelin for cachexia. J Cachexia Sarcopenia Muscle 1: 169-76, 2010

6. Vigano A, Piccioni M, Trutschnigg B, Hornby L, Chaudhury P, Kilgour R: Male hypogonadism associated with advanced cancer : a systematic review. Lancet Oncol 11 : 679-684, 2010

7. Tenover JL: Male hormone replacement therapy including "andropause". Endocrinol Metab Clin North Am 27 : 969-987, 1998

8. Taya M, Koh E, Izumi K, Iijima M, Maeda Y, Matsushita T, Iwamoto T, Namiki M : Comparison of testosterone fractions between Framingham Heart Study participants and Japanese participants. Int J Urol 21 : 689-695, 2014

9. Khosla S, Melton LJ, 3rd, Riggs BL: Clinical review 144 : Estrogen and the male skeleton. J Clin Endocrinol Metab 87 : 1443-1450, 2002

10. Konaka H, Sugimoto K, Orikasa H, Iwamoto T, Takamura T, Takeda Y, Shigehara K, Iijima M, Koh E, Namiki M ; EARTH study group : Effects of long-term androgen replacement therapy on the physical and mental statuses of ageing males with late-onset hypogonadism : a multicenter randomized controlled trial in Japan (EARTH Study). Asian J Androl 18 : 2534, 2016

11. Barrett-Connor E, Goodman-Gruen D, Patay B : Endogenous sex hormones and cognitive function in older men. J Clin Endocrinol Metab 84 : 3681-3685, 1999

12. Christiansen K, Knussmann R: Sex hormones and cognitive functioning in men. Neuropsychobiology $18: 27-36,1987$

13. Shigehara K, Sugimoto K, Konaka H, lijima M, Fukushima M, Maeda Y, Mizokami A, KohE, Origasa H, Iwamoto T, Namiki $\mathrm{M}$ : Androgen replacement therapy contributes to improving lower urinary tract symptoms in patients with hypogonadism and benign prostate hypertrophy : a randomised controlled study. Aging Male 14(1) : 53-8, 2011

14. Shigehara K, Konaka H, Koh E, Izumi K, Kitagawa Y, Mizokami A, Nakashima T, Shimamura M, Iwamoto T, Namiki M : Effects of testosterone replacement therapy on nocturia and quality of life in men with hypogonadism : a subanalysis of a previous prospective randomized controlled study in Japan. Aging Male 18 : 169-174, 2015

15. Kloner RA, Carson C, 3rd, Dobs A, Kopecky S, Mohler ER, $3^{\text {rd }}$ : Testosterone and Cardiovascular Disease. J Am Coll Cardiol $67: 545-557,2016$

16. Izumi $\mathrm{K}$, Chang $\mathrm{C}$ : Targeting inflammatory cytokines-androgen receptor (AR) signalling with ASC-J9(R) to better battle prostate cancer progression. Oncoimmunology 2 : e26853, 2013

17. Fang LY, Izumi K, Lai KP, Liang L, Li L, Miyamoto H, Lin WJ, Chang $\mathrm{C}$ : Infiltrating macrophages promote prostate tumorigenesis via modulating androgen receptor-mediated CCL4STAT3 signalling. Cancer Res 73 : 5633-5646, 2013

18. Izumi K, Mizokami A, Lin WJ, Lai KP, Chang C : Androgen receptor roles in the development of benign prostate hyperplasia. Am J Pathol 182 : 1942-1949, 2013

19. Del Fabbro E, Garcia JM, Dev R, Hui D, Williams J, Engineer D, Palmer JL, Schover L, Bruera E : Testosterone replacement for fatigue in hypogonadal ambulatory males with advanced cancer : a preliminary double-blind placebo-controlled trial. Support Care Cancer 21 : 2599-2607, 2013 